\title{
Germanica
}

\section{Der Dadajsee ruft. Artur Beckers Reisen in die Vergangenheit}

The Call of Lake Dadaj. Journeys into the past in the works of Artur Becker Le Lac Dadaj appelle. Voyages dans le passé dans l'œuvre d'Artur Becker

\section{Ewa Mazurkiewicz}

\section{(2) OpenEdition}

Journals

Édition électronique

URL : http://journals.openedition.org/germanica/2017

DOI : 10.4000/germanica.2017

ISSN : 2107-0784

Éditeur

Université de Lille

Édition imprimée

Date de publication : 31 décembre 2012

Pagination : 165-176

ISBN : 9782913857308

ISSN : 0984-2632

\section{Référence électronique}

Ewa Mazurkiewicz, "Der Dadajsee ruft. Artur Beckers Reisen in die Vergangenheit », Germanica [Online], 51 | 2012, Online erschienen am: 14 Januar 2013, abgerufen am 06 Oktober 2020. URL http://journals.openedition.org/germanica/2017 ; DOI : https://doi.org/10.4000/germanica.2017

Ce document a été généré automatiquement le 6 octobre 2020.

(c) Tous droits réservés 


\title{
Der Dadajsee ruft. Artur Beckers Reisen in die Vergangenheit
}

\author{
The Call of Lake Dadaj. Journeys into the past in the works of Artur Becker \\ Le Lac Dadaj appelle. Voyages dans le passé dans l'œuvre d'Artur Becker
}

Ewa Mazurkiewicz

1 Für den $1968 \mathrm{im}$ polnischen Bartoszyce (Masuren) geborenen Schriftsteller Artur Becker ist sein Polen 1989 untergegangen. Der Systemwechsel und die gesellschaftlichen Transformationen jenseits der Oder bilden zugleich eine Zäsur in seinem Emigrantenleben: Das Land seiner Kindheit existiert für ihn von nun an nicht mehr, ist zu Stein geworden ${ }^{1}$, zu einer sentimentalen persönlichen Geschichte, die es aus der Vergessenheit herauszuholen gilt. Eine literarische Erinnerungsreise in die kleine Heimat Masuren mit ihrem zentralen Punkt am geliebten Dadajsee wird immer wieder zu einer existenzbedingten Aufgabe des Autors.

2 Der folgende Beitrag erschließt das Problem der neuesten deutsch-polnischen Beziehungen aus der interkulturellen Perspektive, indem neben seinen als Grenzgänger erscheinenden Figuren nicht zuletzt auch Beckers Konfrontation mit der Fremde - der deutschen Sprache, Kultur und Geschichte untersucht wird. Darüber hinaus wird den Fragen nachgegangen, welche Position der deutsch schreibende polnische Autor zwischen den beiden Sprachen und Ländern einnimmt, wie er zu dem Phänomen der Emigration steht und wie er mit seiner Vergangenheit umgeht.

3 Artur Becker siedelte, so wie es seine deutsch-polnischen Eltern aus politischen Gründen ein Jahr zuvor getan hatten, 1985 nach Verden an der Aller über. Abgesehen von den wenigen bei der Großmutter gelernten Vokabeln kannte der 17-jährige Immigrant die Sprache des Ziellandes kaum und musste Deutsch als Anfänger lernen. Seine literarische Aktivität in der polnischen Sprache beschränkte sich in seiner Jugendzeit lediglich auf einige Gedichte. Nun wechselte Becker nicht nur die Sprache, sondern auch die literarische Form - er begann Prosa auf Deutsch zu verfassen: „Diese Entscheidung war sehr schmerzlich, weil mir klar wurde, dass ich mich scheiden lasse von der polnischen Literatur und von der polnischen Dame“ ${ }^{{ }^{\prime}}$. In Bremen studierte er 
Kulturgeschichte Osteuropas und Deutsche Literatur- und Sprachwissenschaft, zu dieser Zeit publizierte er auch in deutschsprachigen Zeitschriften Gedichte und Prosafragmente. Sein Debütroman Der Dadajsee erschien 1997 und brachte dem Autor ein Stipendium der Heinrich-Böll-Stiftung und eine Auszeichnung des Deutschen Schriftstellerverbandes ein. Für seine weitere literarische Aktivität erhielt Becker u.a. das Villa-Decius Stipendium in Krakau (1998), Deutsches-Haus Stipendium in New York (2000), Projektförderung der Robert Bosch Stiftung „Grenzgänger - Recherchen in Mittel- und Osteuropa“ (2005), den Adelbert-von-Chamisso-Preis (2009) sowie den DIALOG-Preis 2012 der Deutsch-Polnischen Gesellschaft für seinen Beitrag zur deutschpolnischen Verständigung ${ }^{3}$.

4 Seine Prosatexte sind stark autobiographisch geprägt - die Figuren bewegen sich vorwiegend zwischen Bremen und Masuren, was Becker mit einer Verpflichtung gegenüber der eigenen Vergangenheit begründet: „Ich kann mir nicht vorstellen, dass es etwas Wichtigeres geben könnte als die Autobiographie ${ }^{\text {" }}$. Den Vorwurf, als Schöpfer erstarrt an denselben Orten zu verweilen, entschärft er mit seiner auf Selbstreflexion beruhenden Schreibstrategie: In den Schreibprozess sei nämlich eine Therapie integriert, der Autor erzählt nicht nur dem Leser seine Geschichten, sondern er holt sie aus der Vergessenheit vor allem für sich zurück, um an ihnen halten zu können. Aus der Heimat und deren Zusammenhang herausgerissen, braucht er Distanz, um zu sich selbst und zu den Wurzeln zurückzukommen. Der starke Hang zur Vergangenheitsbewältigung und der ständige Blick auf die masurische Landschaft bleiben nicht ohne Wirkung auf seine deutsche Existenz. Nach so vielen in Deutschland verbrachten Jahren sieht sich Becker dort nur als Gast, trotz Sesshaftigkeit und perfekter Sprachbeherrschung ist die Fremdheit nicht zu löschen: Becker ist und bleibt „polnischer Autor deutscher Sprache“ ${ }^{\text {"5 }}$. Die klare Abgrenzung des Deutschen und Polnischen ist sowohl in seinem privaten, als auch im schriftstellerischen Leben wahrnehmbar: Deutsch sei Beckers „Dienstsprache“, zu Hause spricht er dagegen mit seiner Frau Polnisch, da ihm die Muttersprache nicht zuletzt als Berufsinstrument kostbar ist. Becker und seine Figuren erfahren nun eine doppelte Perspektive, führen ein an Schizophrenie grenzendes Doppelleben als Deutsche und Polen: „Schreibend kommt man aus dieser gebrochenen Identität nicht raus. Der Prozess wird nie abgeschlossen sein" ${ }^{\text {"6 }}$. Als Schriftsteller ist er auch nicht an der deutschen Realität interessiert, dazu fehle ihm die nötige Distanz - die als schreibstrategische Voraussetzung gedachte, lange Inkubationszeit hindert ihn daran, einen Text mit aktuellem deutschem Bezug zu verfassen, darüber hinaus erscheint ihm in Deutschland nichts schreibenswert ${ }^{7}$, denn auch Nostalgie und Melancholie erkennt er als entscheidende Faktoren und Antriebskraft für den Schreibprozess.

Die Dualität eines zwischen zwei Sprachen und zwei Nationen lebenden Menschen bekennt Becker selbst und überträgt dieses sich bald glückbringend, bald verhängnisvoll ausgebende Gefühl auf seine Helden. Zerrissen zwischen Ost und West, finden sie einen Ort dazwischen - ähnlich, wie die Figuren der anderen auf Deutsch schreibenden Emigranten Wladimir Kaminer und Radek Knapp - sie emanzipieren sich von dem Osten, der Westen bleibt ihnen jedoch immer fremd. Die Gegensätze und Reibungen, das Hin und Her der Beckerschen Grenzgänger und Außenseiter spiegeln sich auf jeder Ebene seines Textes wider: die ständig schwankenden Figuren, die nirgendwo ankommen können, deren Gemütslage zwischen Glück und Depression, Nähe und Distanz, Eros und Thanatos balanciert, erscheinen vor dem Hintergrund der sie umgebenden Natur - einer Gewalt, die jene Gegensätze noch zu verstärken vermag. 
Wie alle Erzählelemente bei Becker funktioniert auch die Natur nach dem Prinzip des Kontrapunkts. Zum einen gilt hier die idyllische masurische Landschaft als Gegenpol der hässlichen, grauen Ästhetik des Sozialismus, zum anderen ist sie jedoch selbst ein dualistisches Phänomen - sie ist gut und böse zugleich, schön und gefährlich für den Menschen, der ihr respektvoll zu begegnen hat: „Die schöne Natur birgt nämlich auch Unglaubliches und Gefährliches in sich. Aus der Verwunderung darüber konnte ich nur Schriftsteller werden“", meint Becker. Durch die Darstellung der gefährlichen, den Menschen bedrohenden Natur greift Becker auf einen wichtigen Topos in der polnischen Literaturtradition zurück und integriert ihn somit in die neueste deutsche Literatur.

6 Aus der doppelten Perspektive versucht der Schriftsteller auch auf beide Länder, deren Geschichte und Gegenwart, zu blicken, wobei Vergleich und Konfrontation kaum zu vermeiden sind. Das Leben fern von der Heimat ermöglicht ihm eine Distanz zu deren Politik und Geschichte, lässt dagegen die Geschichte des Gastlandes aufmerksamer reflektieren. Trotzdem bleibt ihm die deutsche Geschichte fremd, da er kein Teil von ihr sei. Die kulturellen Unterschiede zwischen Polen und Deutschland nimmt Becker besonders im existentiellen Bereich wahr: „Das Leben im Westen vollzieht sich weit entfernt von den großen Themen, der Tod etwa ist ein solches Tabu, dass ich nur aufschreien kann".9. Aufgewachsen in einem streng katholischen Land, wo etwa ein Trauerzug nicht nur mit dem Glauben, sondern auch mit der Folklore verbunden ist und wo die Alten geachtet werden, begegnet der Autor solchen zivilisatorischen Veränderungen wie der Tabuisierung des Todes und des Alters in der säkularisierten deutschen Gesellschaft eher mit Distanz und Misstrauen.

7 Zwar richtet sich Beckers literarische Aufmerksamkeit auf seine eigene Geschichte, das aktuelle Geschehen in Polen bleibt in seinen neuesten Texten aber auch nicht ohne Beachtung. Als Autor und Privatperson nimmt er Stellung etwa zu dem EU-Beitritt Polens und vergleicht die Bindung seiner Heimat an Brüssel mit der früheren Bindung an Moskau: „Durch die EU haben wir eine Art Zentralregierung bekommen, die uns Polen bekannt ist aus sozialistischer Zeit. Manche spotten: Früher fuhr man nach Moskau, um Befehle zu holen, jetzt fährt man nach Brüssel oder Washington “"10. Auf der anderen Seite erinnert er sich an die dunklen Zeiten der sozialistischen Diktatur in Polen und meint, daraus eine wichtige Lehre gezogen zu haben: nach der Erfahrung und Überwindung des Regimes könne man nur als Optimist in die Zukunft blicken, auch wenn der heutige Mensch allerlei wirtschaftlichen und moralischen Krisen ausgesetzt ist - nach jedem Tief kommt irgendwann ein Hoch. Aus der zeitlichen und räumlichen Distanz beurteilt Becker auch die bis heute umstrittene Persönlichkeit des sozialistischen Generals und Ex-Präsidenten Jaruzelski, der die Hauptverantwortung für die Verhängung des Kriegsrechts in Polen trägt, dessen Schuld bis heute vor polnischem Gericht und von der Gesellschaft erörtert wird. Es mag auch an der interkulturellen Perspektive liegen, dass sich Becker an die Seite der gar nicht geringen Zahl der Verteidiger Jaruzelskis mit der Begründung stellt, das damalige Staatsoberhaupt habe Polen vor einer militärischen und wirtschaftlichen Katastrophe gerettet. Ganz entschieden wendet sich Becker jedoch nicht nur gegen die Beteiligung polnischer Soldaten am Irak-Krieg, sondern gegen die gesamte Amerika-Initiative im Irak.

8 Viele von den auf Polens Vergangenheit und Gegenwart bezogenen Themen finden ihre Widerspiegelung in dem 2008 veröffentlichten Roman Wodka und Messer: der ehemalige 
Funktionär der Staatssicherheit und Mörder einer der Figuren bekleidet heute das Amt des Bürgermeisters, mit Hilfe der zweideutigen Figur des zum Katholizismus konvertierten Rabbi wird die in Polen immer noch aktuelle Frage des Antisemitismus aufgeworfen, nicht ohne Ironie erscheint hier Polen in der neuen Rolle als EU-Mitglied, aus dem Mund einer der Figuren kommt die Empörung über die polnische militärische Mission im Irak. Der Protagonist Kuba dagegen verkörpert am deutlichsten von allen Helden - und zwar wortwörtlich - den Beckerschen Dualismus und die doppelte Perspektive - er wird Zweibauchnabel genannt, weil in seinem Bauch sein toter Zwillingsbruder entdeckt wurde und ihn immer wieder als ein zweites Ich anspricht. Als eine der wenigen Figuren unter Beckers Emigranten gehört Kuba zu den in Deutschland Sesshaften im positiven Sinne - er lebt dort mit seiner Familie, hat eine gute Arbeit, doch der heimatliche Dadajsee - die Vergangenheit - ruft ihn eines Tages zurück nach Masuren, wo seine Jugendzeit und seine Geschichte geblieben sind. Somit beginnt für den Helden eine teils glückbringende, teils schmerzhafte Reise in die Kindheit, aber auch zugleich in die Gegenwart. Kuba wird mit zwei Welten konfrontiert - einerseits sucht er nach den Spuren seiner eigenen Geschichte, andererseits begegnet er seiner Heimat in ihrer neuen Haut und mit ihren aktuellen Problemen. Doch die zentrale Funktion übernimmt, wie immer bei Becker, der Dadajsee als Metapher der Kindheit, die Verkörperung der Sehnsucht nach der masurischen Heimat. Die Helden kommen entweder freiwillig oder gezwungen, wie etwa Jurek aus dem 1997 erschienenen Roman Der Dadajsee, auf den im Folgenden ausführlicher eingegangen wird, zu dem See und den Menschen in dessen Umgebung, um die seit Jahren offene Rechnung zu begleichen - bei den anderen, jedoch vorwiegend bei sich selbst. Erst am Ufer des Dadajsees, ob sie ihn lieben oder hassen, kommen sie zu der Erkenntnis, dass sie in ihrem Pendeln zwischen Ost und West eigentlich ortlos sind, nirgendwo fest verankert, wie die Boote, die sich ständig zwischen zwei Ufern bewegen.

Obwohl Beckers Figuren im deutschen Raum keinen Halt finden können, sind ihre Beziehungen $\mathrm{zu}$ den Einheimischen frei von klischeehaften Antagonismen und Xenophobie. Nicht auf den Umgang mit den Deutschen kommt es bei den Helden an, sondern auf die individuelle Anpassungsunfähigkeit so im Osten wie im Westen. Becker richtet den Fokus zwar meistens auf einen problematischen Ausländer, das Problem stellt jedoch vor allem dessen Randexistenz an sich dar. Sowohl Polen als auch Deutsche erscheinen in Beckers Texten nicht selten als Verlierer, Außenseiter und Lebenskünstler, der Autor verzichtet bewusst auf nationalbedingte Menschenkategorisierung, denn er sieht die Emigration vielmehr als „eine gute Form, um zu einem kosmischen oder eben universellen Menschen zu werden. Also zu einem 'Erdling ““11. Jede Zuordnung der Immigranten zu ihrer Nationalität beeinträchtigt laut Becker die zwischenmenschliche Kommunikation, da man zuerst Mensch ist, erst im Umfeld der nationalen Zugehörigkeit entstehen dagegen Vorurteile und klischeehafte Verhaltensmuster, die eine unvoreingenommene Begegnung innerhalb der multikulturellen Gesellschaft verhindern.

10 Von den acht innerhalb von fünfzehn Jahren veröffentlichten Romanen über Aufbruch und Rückkehr wird im Folgenden Beckers Erstling mit dessen multidimensionaler Welt zwischen Ost und West näher betrachtet. Der Roman Der Dadajsee ist 1997, nur wenige Jahre nach dem Systemwechsel in Polen erschienen, die Handlung spielt sich gerade um 1990 in Bremen und Masuren ab. Die Wende spiegelt sich im Text kaum wider, das Geschehen konzentriert sich nämlich auf Menschen, die sowohl im Osten als auch im 
Westen weit von Politik und aktuellen Tagesereignissen leben, deren Anliegen zwischen Mensch und Natur situiert ist.

11 Der Hauptheld Jurek emigriert kurz nach der Einführung des Kriegsrechts in Polen aus seiner masurischen Gegend nach Bremen. Der Zeitpunkt seiner Flucht mag irreführend sein, denn er motiviert seine Entscheidung weder politisch noch ökonomisch, sondern manifestiert auf diese Weise die Auflehnung gegen den elterlichen Willen. Sein Vorhaben, nach dem Abitur ein Studium aufzunehmen, steht nämlich im Widerspruch zu den Plänen seines Vaters - der Sohn solle den Bauernhof übernehmen. Hasserfüllt verlässt Jurek sein Zuhause, um in Deutschland ein Immigrantenleben zu führen. Die Handlung spielt anfangs parallel in Bremen und Masuren, dem Leser werden die beiden Welten wie Gegenpole vor Augen geführt, bis der Höhepunkt eintritt, wenn Jurek dazu verleitet wird, sein Elternhaus wieder zu besuchen. Im Gegensatz zu Beckers anderen Figuren (Kino Muza, Wodka und Messer, Onkel Jimmy, die Indianer und Ich u.a.) wird Jurek nicht von der Sehnsucht nach der Heimat getrieben, sondern muss sich überwinden, nach dem Tod des Vaters den scheinbar endgültig abgeschlossenen Lebensraum nochmals zu betreten. Nachdem er seine Familie und seine Heimat aufgegeben hat, kommt er jedoch in dem Gastland auch nicht richtig an - zu seiner Wohnung wählt er einen Wohnwagen im Garten seines Freundes, des reichen Psychiaters Harri: „Jurek hätte auch in der Villa leben können, [...] aber Jurek lebte die Freiheit, das Freie, die Luft. Am liebsten würde er in einem Zelt leben, im Wald, am See, ohne Dusche und Küche. Im Zentrum der Stadt, in den Einkaufspassagen knöpfte er immer das Hemd bis zum Bauch auf und betastete seinen Hals, weil er sich einbildete zu ersticken“ (113). Die Stimme des in ihm steckenden Naturmenschen vom Dadajsee versucht Jurek im Stadtleben zu betäuben, instinktiv sucht er jedoch nach Orten, die ihm seine Freiheit gewährleisten und die masurische Wildnis kompensieren. Der Wohnwagen steht auch symbolisch für eine vorübergehende Station, der polnische Junge ist in Deutschland nicht fest verankert und nicht zu Hause - er ist ort- und heimatlos, seine Existenz schwebt in einem Zwischenraum, nicht mehr in Polen und noch nicht in Deutschland. Eine Stabilität strebt Jurek in der Emigration jedoch nicht an, er schließt eine Scheinehe mit der Schauspielerin Kornelia, um das Bleiberecht für Deutschland zu erwerben - eine lockere Beziehung ohne Verpflichtungen: „sie liebten sich in Wirklichkeit sehr, doch nur in bestimmten Augenblicken, nicht jeden Tag, sie benahmen sich wie Quartalsäufer, sie waren ein bisschen süchtig, aber von der Leidenschaft keineswegs verblendet, sondern aufeinander neugierig - ein Zustand des ständigen Neubeginns war es, und nur das zählte“ (118). Seinem Studiumvorhaben bleibt Jurek auch nicht treu, kurz vor dem Abschluss bricht er das Soziologiestudium ab, um als Erzieher und Nachtwächter zu arbeiten.

12 Nach dem mutigen Ausbruch aus dem Heimatland, bei dem die Elternliebe aufs Spiel gesetzt wurde, muss sich Jurek nun in der Fremde als einen Verlierer betrachten, der für sich auch in Polen keinen Neuanfang mehr zu sehen glaubt. Sobald ihm jedoch eine Reise an den Dadajsee in Aussicht gestellt wird, gerät er in Verwirrung, wird unruhig, und aus seinem Unterbewusstsein steigen Bilder aus der Vergangenheit empor: „diese Sonne des Sommers, in der er seine Mutter zu sehen glaubte, die letzte Filmaufnahme, kein Gedanke in Worten, wirklich gesprochen, nein, sondern nur die Mutter von hinten auf dem Sandweg zum Dadajsee, [...] aber die Bilder, die Schappschüsse, der See und die Felder bedrohten Jurek nicht, sie flossen nur an ihm vorbei“ (131). So sehr er die schmerzliche Vergangenheit auch aus seinem Gedächtnis zu verdrängen versucht, sie lässt sich nicht auslöschen. Von den Bildern aus der Jugendzeit zu Hause sowie den 
gegen den Vater gerichteten vorwurfsvollen Gedanken wird er wider Willen nahezu überfallen. In einer schlaflosen Nacht von plötzlichen Todesgedanken gequält, verspürt er gleichzeitig einen starken Drang nach sexuellem Erleben. Er wird von Eros und Thanatos zugleich getrieben, auch selbst in Momenten der erotischen Ekstase: „Ich fick dich tot, schrie Jurek Kornelia ins Ohr" (130), um kurz danach wieder an die bevorstehende Reise denken zu müssen: „Er stellte sich vor, wie er die Pforte öffnete, den Bauernhof seiner Eltern betrat, er stellte sich vor, wie lächerlich es sein würde, der Mutter Guten Tag zu sagen, nach so vielen Jahren“(131f).

13 Jurek fährt in sein Elternhaus nicht um der Versöhnung willen - trotz des Todes will er mit dem Vater noch abrechnen und sich somit endgültig von ihm befreien ${ }^{12}$. Die Ablösung von dem verhassten Vater soll durch dessen Enthauptung zustande kommen: „Ich werde die Leiche meines Vaters ausgraben, ich werde seinen verdammten Kopf abschneiden“ (147). Der Vater-Sohn-Konflikt ist so tief in Jurek verwurzelt, dass er erst dann zur Ruhe kommen kann, nachdem er selbst das Todesritual vollbracht hat. Dabei stößt er auf Unverständnis bei Kornelia, die er in diesen Generationskonflikt und in die gesamte polnische Vergangenheit nicht eingeweiht $\mathrm{zu}$ haben scheint, die beiden Welten bleiben voneinander getrennt, seine Gegenwart ist mit der Geschichte nicht zu vereinigen: „Kornelia versteht mich nicht, sie wird es nicht verstehen, für sie ist das nur eine Erholungsreise, reine Neugier, wie es aussehen mag, dort, wo ihr Mann, der gute Jurek, die Hälfte seines Lebens verbracht hat" (149). Die Reise nach Polen bringt den Protagonisten wiederum in einen Zwiespalt - ein Gefühl, das ihn sowohl im Westen als auch im Osten begleitet. Im Elternhaus angekommen, erwacht er nachts mit dem Gedanken, ob jener Ort noch als sein Zuhause zu nennen sei, „eigentlich war er sich überhaupt nicht sicher, wo sein Zuhause war" (149).

Eine Art Konfrontation mit der Welt seiner Kindheit erfolgt über die hautnahe Berührung mit deren Sinnbild - dem Dadajsee. Jurek taucht in ihn bei Mondschein ein, als würde er sich auf eine harte Probe stellen, ob etwa dort noch seine unversehrte Jugendzeit wiederzufinden sei. Doch der Zauber aus der Vergangenheit wirkt nicht mehr: „Dadaj. Er verstand diesen Namen nicht mehr, der Klang dieses Wortes war für ihn fremd geworden, ein fremdes Wort, der Dadajsee, dachte er" (150). Trotzdem kann ihm der See Erleichterung schenken, er vergisst für einen Augenblick alle seine Sorgen und freut sich, von der Natur umgeben zu sein. Nun wieder kämpfen in Jureks Seele widersprüchliche Elemente - Natur und Kultur -: einerseits will er dem symbolischen, nahezu heiligen Dadajsee respektvoll gegenüberstehen, andererseits lässt ihn die Erinnerung an die Jugendzeit, an den als Fischer tätigen Vater, der den See und die Fische mehr liebte als den eigenen Sohn, dem See nur noch Hass entgegenbringen. Er kann sich mit dem Land seiner Eltern nicht mehr identifizieren und nicht mehr versöhnen - zu der Erkenntnis kommt er, indem er sich in dem Dadajsee wie im Spiegel betrachtet: „welche Stimme ruft aus dem Dadajsee? Nicht meine Stimme, ich hasse diesen See, kam ihm plötzlich in den Sinn, ich hasse ihn, weil er mich zum Schwächling hat werden lassen, ich bin zu schwach, um in der Welt zu überleben“ (176). Diese bittere Katharsis, bekräftigt durch den Akt der Versenkung des abgeschnittenen Kopfes seines Vaters im Dadajsee, erlaubt dem Helden, sich auf die Suche nach seinem Lebensort zu begeben, den er vielmehr emotional als topographisch festzulegen versucht. Die Lücke nach der verlorenen Heimat scheinen seine Frau Kornelia und die beiden deutschen Freunde zu füllen, deren Nähe Jurek während des Polenaufenthalts mehr begehrt, als die Begegnung mit seiner Mutter. Obwohl diese bereit ist, Jurek wie den biblischen verlorenen Sohn, der tot war und wieder lebendig geworden ist, mit 
offenen Armen wieder aufzunehmen, meidet er jegliche direkte Konfrontation mit ihr und bewahrt kühle Distanz: „Ich weiß nicht, wer du bist, sagte Jurek, ich weiß nicht einmal, wer ich bin“ (156). Mit Ausnahme der Annäherung an den Dadajsee verweigert er jeden versöhnlichen Schritt zurück in die Welt der Jugendzeit, um das Ziel nicht zu verfehlen, mit dem er seine Reise zu den Wurzeln angetreten hatte: Abrechnung und Ablösung. Das gelingt ihm auch.

Jureks Existenz in Deutschland ist nur in geringem Maß an den Raum gebunden, er sucht bei dem westlichen Nachbarn weder hohen materiellen Status noch politischen Schutz, wie es noch seine Landsleute - die mittelosteuropäischen Dissidenten - eine Dekade früher anstrebten. Becker schafft einen neuen Heldentypus, frei von klischeehaftem Bild eines den Deutschen immer fremden Immigranten und Gastarbeiter. Den Halt findet Jurek gerade unter deutschen Freunden, die, ähnlich wie er selbst, ein entspanntes Dasein führen. In dem Milieu von Gleichgesinnten braucht der polnische Ausreißer nichts zu beweisen, er wird vor allem als Mensch und Partner nicht als ausländischer Außenseiter - behandelt. Bei Harri, einem reichen Psychiater, der in einer vom Großvater geerbten Luxusvilla wohnt, findet Jurek nicht nur eine Wohnung, sondern auch eine richtige Freundschaft. Es ist auch Harri, der den polnischen Freund in sein an der Grenze der Legalität liegendes Geschäft einweiht: Er kauft von einem psychiatrischen Krankenhaus in Deutschland kurz vor dem Verfallsdatum stehende Medikamente und verkauft sie mit hohem Gewinn an polnische Krankenhäuser und Apotheken. Für den Transport macht er Jurek verantwortlich. Die Männer verbringen ihre Freizeit bei Wein, Joints und Musik - vor allem von Keith Jarrett. Als Jurek die Reise nach Polen ankündigt, zögert Harri keinen Moment, um dem Freund seine Begleitung anzubieten. Zu Jureks engem Freundeskreis gehört auch der Kneipenbesitzer Moogi, der in der Freizeit Autos repariert: „Seine Kunden? Die Arbeitslosen, die Studentinnen, die Entenfahrer, die Zauberer, Künstler, die von der Hand in den Mund leben - die Mehrheit von diesen Leuten war nicht älter als dreißig“ (106). Auch Moogi fragt nicht nach Jureks Nationalität, für die beiden zählt menschlicher und freundschaftlicher Umgang miteinander, der in der Ära des Wettbewerbs eine Seltenheit darstellt: „Schon am ersten Tag bemerkte Jurek, dass dieser Typ, der sich mit dem Namen Moogie vorgestellt hatte, keiner Fliege etwas zuleide tun konnte, das war in seinem Gesicht zu sehen, diese Gutmütigkeit und Offenheit, die Jurek selten bei anderen Leuten spürte“ (106). Sowohl mit Moogi als auch mit Harri verbindet Jurek eine große Liebe zu Musik, besonders von Miles Davis, Keith Jarret und Pink Floyd, sie begleitet ihre freundschaftlichen Treffen und wird zum Gesprächsthema über alle nationalen, politischen und kulturellen Grenzen hinweg. Die Musik erweist sich für die in den 60-er Jahren geborene Generation als ein interkulturelles Bindungselement und verhilft aufgrund ihrer Universalität den jungen Menschen $\mathrm{zu}$ ungestörten Beziehungen mit den Fremden: „Moogi hatte immer irgendwelche Kassetten dabei; gute Musik sorgt stets für gute Laune, das war sein Motto, danach lebte er" (105). Jurek, ein Musik und Alkohol liebender Junge aus dem Ostblock, findet somit sofort einen Anschluss an die gleichgesinnten Seelen in der scheinbar verschlossenen Welt von fremder Sprache und Kultur. Mehr als beruflichen Erfolg und eine etablierte Existenz in Deutschland schätzt er seine Freiheit und Befriedigung unmaterieller Bedürfnisse: „Kornelia, ich verdiene in meinem Job nicht viel, das weißt $d u$, außerdem brauche ich viel Zeit für mich, fürs Lesen und Musikhören“ (116). 

gehört, scheint wie eine gegenwärtige Hippie-Gemeinschaft zu leben, in der neben Sex, Drugs und Rock'n Roll vor allem ein unbekümmertes, erlebnisorientiertes Dasein zählt. Insofern erinnert Beckers Text an den 1998 von Judith Hermann veröffentlichten Erzählband Sommerhaus später, dessen Figuren - vorwiegend passive Außenseiter - ein bohemehaftes Leben führen und von einer Entscheidungsimpotenz betroffen sind. So wie Beckers Gestalten existieren sie außerhalb der offiziellen gesellschaftlichen Ordnung und Normen. Moogi, Harri und Kornelia gehen zwar ihren Berufen nach, die Arbeit steht hier aber nicht im Mittelpunkt ihrer Existenz. Hermanns und Beckers (Anti)Helden gehören zu einer unpolitischen, melancholischen Generation, die ein Leben jenseits der konsum- und leistungsorientierten Welt führt. Unter diesen Umständen fällt es dem polnischen Immigranten Jurek nicht allzu schwer, sich in die deutsche Gesellschaftsnische einzuleben - so wie seine Freunde verspürt er keinen Drang, sich im Leben zu etablieren, sondern wird von ihnen zur Freiheit ermuntert, wie etwa durch die Scheinehe ohne feste Regeln oder das Verbleiben im provisorischen Wohnwagen. Der Protagonist scheint in permanenter Adoleszenz zu stehen, nach der Flucht aus Polen sieht er sich von jeglichen Verpflichtungen befreit, auch vom Erwachsenwerden, und wird von der Umgebung auch nicht dazu motiviert - Moogi, Harri und Kornelia leben ihm als Einheimische solche Haltung vor. Die Letztere versucht ihn doch mit der Zeit zu einem verantwortungsbewussten Dasein zu verleiten, indem sie ihm seine gefährlichen Geschäfte mit Medikamenten sowie das abgebrochene Studium zum Vorwurf macht. Auf Moogi, Harri und Kornelia ist Verlass - Jurek hätte sich in Polen keine besseren Freunde vorstellen können -, und trotzdem kann er in der Emigration nicht Fuß fassen. Das Problem liegt nämlich bei ihm selbst, nicht an der Sprache, den Kulturdifferenzen, dem Aufenthaltsort und nicht am politischwirtschaftlichen System: „Der Aufenthalt im Westen ist ein auf Dauer gestellter Transit ${ }^{{ }^{\prime 13}}$.

Jureks Aufenthalt in Deutschland verändert ihn nicht allzu sehr. Zwar lernt er bei Kornelia, den Wein zu genießen, statt sich zu betrinken, wie er es in Polen zu tun pflegte: „In Masuren hatte er fast jeden Tag getrunken, Wodka und Bier, so wie sein Freund Ludwik, mit dem Saufen hörte er nach der Auswanderung nach Deutschland auf, hier erst entdeckte er den Kaffee, der die Herzschläge beschleunigte, den Kopf aber nüchtern und klar ließ“ (115). Als einem Jungen aus der masurischen Provinz wird ihm auch ein Arztbesuch zu einer ungewöhnlichen Erfahrung - an dieser Stelle zeigt Becker die zivilisatorischen Unterschiede zwischen Ost- und Westeuropa, besonders die Defizite im sozialen Bereich: Jurek besuchte nie einen Zahnarzt, stattdessen „kaufte er sich eine Zeitung, riss ein Stück von ihr ab und stopfte den Fetzen in das Loch im Zahn. Die Tageszeitungen eigneten sich sehr gut dazu, weil ihre Farbe viel wirksamer war als die der Illustrierten“ (105).

Obwohl Beckers Figuren im interkulturellen Kontext nach einem ähnlichen Modell aufgebaut sind - als junge Emigranten aus Polen, die zwischen Ost und West leben und sich allmählich auf einen Höhepunkt zu bewegen, der sie über die Grenze hin oder her gehen lässt -, erweist sich die innere Struktur der Heldentypen jeweils als wesentlich unterschiedlich. Während Jurek - ein Hedonist und Gelegenheitsarbeiter - sich mit der bescheidenen Existenz in Deutschland zu begnügen versucht und der Heimat den Rücken zeigt, geht etwa Antek Haack - der Protagonist des 2003 veröffentlichten Romans Kino Muza - in seinem deutsch-polnischen Abenteuer pragmatisch vor. Als 
Kartenabreißer im Kino Muza, angesiedelt im masurischen Bartoszyce, nimmt er jedes Jahr unbezahlten Urlaub, um in Deutschland als Anstreicher Geld zu verdienen. Im sozialistischen Polen der 80-er Jahre erscheint ihm diese Verdienstmöglichkeit als die einzige Chance, seinen Traum zu verwirklichen, zusammen mit einem Freund dem Staat das Kino Muza abzukaufen. Anders als Jurek wird er in seiner Jugendzeit direkt mit dem kommunistischen Regime und dessen Bürokratie konfrontiert, auf deren Kollaps er geduldig wartet, ohne vorschnell - so wie viele andere Bürger - die Flucht in den Westen zu ergreifen. Antek fühlt sich an die heimatliche Gegend, das Elternhaus und seine polnischen Freunde gebunden, doch wenn die Probleme mit der Staatssicherheit sich als lebensbedrohlich erweisen, rettet er sich mit einer endgültigen Flucht nach Deutschland. Im Gegensatz zu dem nahezu kosmopolitisch gesinnten Jurek kann sich Antek weder von seinem Herkunftsland emanzipieren, noch in Deutschland Fuß fassen, seine Existenz konzentriert sich auf Materielles - er lebt in Bremen bei der reichen Lucie, während in Polen zwei andere Frauen ihn begehren. Kuba dagegen - der Held aus Wodka und Messer stellt Jureks völligen Gegensatz dar - seine ersehnte Reise nach Polen ist von Sehnsucht und Liebe nach den dort zurückgelassenen Menschen und der Landschaft um den Dadajsee erfüllt. Nostalgie und Melancholie treiben ihn aus dem Westen in die Welt der Vergangenheit, um nach zwanzig Jahren die Jugendzeit wieder $\mathrm{zu}$ erleben und $\mathrm{zu}$ verstehen - mit ihren Schmerzen und Hoffnungen. Dem in Deutschland zum reifen Mann und Familienvater gewordenen Kuba fehlt Jureks Zorn, eine Rückkehr hält er jedoch ebenfalls für unmöglich - er möchte zurückkehren, aber es geht nicht mehr. Keiner von Beckers in der deutschen Emigration lebenden Ausreißern kann jedoch weder seine tief im Unterbewusstsein schlummernde Unruhe stillen, noch den ewigen Konflikt zwischen dem Fremden und dem Eigenen, unabhängig von Lebensstatus und Situierung innerhalb des deutschen Kulturraumes.

\section{NOTES}

1. Vgl. Karin Großmann: „'Ich habe schon eine Krise überlebt.' Der Autor Artur Becker spricht über sein doppeltes Leben als Deutscher und Pole“, in: Sächsische Zeitung, Wochenmagazin, 1.11. 2008, hier nach: www.arturbecker.de.

2. Artur Becker, in: Vladimir Balzer: „Zwischen den Welten. Der Schriftsteller und Musiker Artur Becker", Deutschlandradio Kulturo. 05.03.2009.

3. Vgl. dazu Homepage von Artur Becker: www.arturbecker.de.

4. Artur Becker, zitiert nach Ingrid Reichel: "Kraft der Erinnerung aus der Kindheit", in: etcetera, 2009, Nr 35, hier:. www.arturbecker.de.

5. Vladimir Balzer, a.a.O.

6. Artur Becker, zitiert nach Karin Großmann, a.a.O.

7. Vgl. Karin Großmann, a.a.O.

8. Artur Becker, in: Jens Nommel: „Artur Becker im Gespräch über Masuren, Zeitreisen in die Kindheit und die Angst vor der Natur“, in: Handlungsreisen.de, 29.09.2008.

9. Artur Becker, zitiert nach Karin Großmann, a.a.O.

10. Artur Becker, ebd. 
11. Artur Becker, zitiert nach Ingrid Reichel, a.a.O.

12. Vgl. dazu auch: Hans Dieter Grünefeld: „Vatermord“, in: Buchkultur. Das internationale Magazin aus Wien, 1998, Nr. 50/1.

13. Jörg Plath: „Knapp, Kaminer, Becker. Drei deutschsprachige Schriftsteller mit polnischem und russischem Hintergrund“, in: Dialog, 2004, Nr. 68, S. 100.

\section{RÉSUMÉS}

Der folgende Beitrag erschließt aus der interkulturellen Perspektive das Problem der neuesten deutsch-polnischen Beziehungen am Beispiel der ausgewählten Texte des auf Deutsch schreibenden polnischen Autors Artur Becker. In seinen autobiographischen Romanen, deren Handlung zwischen Bremen und Masuren situiert ist, betrachtet er das Phänomen der Emigration, der Flucht „ins Paradies“ und der Rückkehr in die Heimat. Beckers Figuren, so wie auch er selbst, erfahren eine Existenz an der Grenze von zwei Sprachen, Kulturen und Geschichte von zwei Nationen. Mit der Zeit wird das Leben jener Ausreißer von einer doppelten Identität dominiert - einem Verlorensein zwischen zwei Welten, zwischen Ost und West. Beckers Helden kommen in einem wichtigen Moment ihres Lebens ins masurische Land zurück, an den Dadajsee, um dort ihre verlorene Identität wiederzufinden und den Menschen sowie der Natur näherzukommen.

Cette étude aborde le problème des relations polono allemandes actuelles d'un point de vue interculturel à partir de l'exemple d'Artur Becker - auteur polonais qui écrit en allemand. Dans ses romans autobiographiques, dont l'action se situe entre Brême en Allemagne et la Mazurie, sa région natale, l'auteur analyse les phénomènes d'immigration, de la fuite vers le « Paradis » et du retour à la patrie. Les personnages, comme l'auteur lui-même, vivent leurs vies à la frontière entre deux langues, deux cultures et l'histoire de deux nations. Leur vie est progressivement dominée par le sentiment d'une double identité et l'errance entre deux mondes - l'Est et l'Ouest. Les héros de Becker reviennent souvent dans leur région natale, au bord du lac Dadaj de Mazurie, afin de retrouver leur identité perdue et renouer contact tant avec les hommes qu'avec la nature.

The present paper discusses contemporary Polish-German relations from an intercultural perspective exemplified by the works of Artur Becker, a Polish author writing in German. His autobiographical novels, set between German Bremen and his native Masuria, touch upon questions of contemporary emigration, of an "escape to paradise" followed by the return to one's own homeland. Becker's characters mirror his own fate and experience life at the crossroads between languages, cultures and histories of the two nations. Their lives are dominated by a growing awareness of dual identity and of being lost and suspended between two worlds, the East and the West. Becker's hero often returns to his native soil by the Masurian Lake Dadaj to reestablish his missing identity and to restore forgotten ties with people and nature.

\section{INDEX}

oeuvrecitee Der Dadajsee 
AUTEURS

EWA MAZURKIEWICZ

Universität Katowice (Polen) 